International Journal of Pure and Applied Mathematics

Volume 101 No. $1 \quad 2015,83-86$

ISSN: 1311-8080 (printed version); ISSN: 1314-3395 (on-line version)

url: http://www.ijpam.eu

doi: http://dx.doi.org/10.12732/ijpam.v101i1.8

ijpam.eu

\title{
SUBSPACE TRANSITIVITY OF TUPLES OF OPERATORS
}

\author{
Bahmann Yousefi ${ }^{1}$, Elham Fathi ${ }^{2}$ \\ ${ }^{1,2}$ Department of Mathematics \\ Payame Noor University \\ P.O. Box 19395-3697, Tehran, IRAN
}

\begin{abstract}
In this paper, we introduce subspace hypercyclicity and transitivity of tuples of operators and we give some relations between these concepts and the subspace transitivity criterion for a tuple of operators.
\end{abstract}

AMS Subject Classification: 47B37, 47B33

Key Words: tuple, subspace hypercyclicity, subspace transitivity, hypercylicity criterion

\section{Introduction}

By an $n$-tuple of operators we mean a finite sequence of length $\mathrm{n}$ of commuting continuous linear operators on a Banach space $X$.

Definition 1.1. Let $\mathcal{T}=\left(T_{1}, T_{2}, \ldots, T_{n}\right)$ be an n-tuple of operators acting on a separable infinite dimensional Banach space $X$ over $\mathbb{C}$ and let $M$ be a nonzero subspace of $X$. We will let

$$
\mathcal{F}=\left\{T_{1}^{k_{1}} T_{2}{ }^{k_{2}} \ldots T_{n}{ }^{k_{n}}: k_{i} \geq 0, i=1, \ldots, n\right\}
$$

be the semigroup generated by $\mathcal{T}$. For $x \in X$, the orbit of $x$ under the tuple $\mathcal{T}$

Received: January 31, 2015

(C) 2015 Academic Publications, Ltd.

$\S$ Correspondence author 
is the set $\operatorname{Orb}(\mathcal{T}, x)=\{S x: S \in \mathcal{F}\}$. A vector $x$ is called a subspace-hypercyclic (or $M$-hypercyclic) vector for $\mathcal{T}$ if $\operatorname{Orb}(\mathcal{T}, x) \cap M$ is dense in $M$ and in this case the tuple $\mathcal{T}$ is called subspace-hypercyclic for $M$. The set of all $M$-hypercyclic vectors of $\mathcal{T}$ is denoted by $H C(\mathcal{T}, M)$. Also, for all $k \geq 2$, by $\mathcal{T}_{d}^{(k)}$ we will refer to the set of all $k$ copies of an element of $\mathcal{F}$, i.e.

$$
\mathcal{T}_{d}^{(k)}=\left\{S_{1} \oplus \ldots \oplus S_{k}: S_{1}=\ldots=S_{k} \in \mathcal{F}\right\} .
$$

We say that $\mathcal{T}_{d}^{(k)}$ is subspace-hypercyclic, with respect to $M$, provided there exist $x_{1}, \ldots, x_{k} \in X$ such that $\left\{W\left(x_{1} \oplus \ldots \oplus x_{k}\right): W \in \mathcal{T}_{d}^{(k)}\right\} \cap M$ is dense in the $k$ copies of $M, M \oplus \ldots \oplus M$.

Note that if $T_{1}, T_{2}, \ldots, T_{n}$ are commutative bounded linear operators on a Banach space $X$, and $\left\{m_{j}(i)\right\}_{j}$, is a sequence of natural numbers for $i=1, \ldots, n$, then we say $\left\{T_{1}^{m_{j}(1)} T_{2} m_{j}(2) \ldots T_{n} m_{j}(n): j \geq 0\right\}$ is $M$-hypercyclic if there exists $x \in X$ such that $\left\{T_{1}^{m_{j}(1)} T_{2}{ }^{m_{j}(2)} \ldots T_{n}^{m_{j}(n)} x: j \geq 0\right\} \cap M$ is dense in $M$.

Definition 1.2. Suppose that $\mathcal{T}=\left(T_{1}, T_{2}, \ldots, T_{n}\right)$ is an n-tuple of operators acting on a separable infinite dimensional Banach space $X$ over $\mathbf{C}$ and $M$ is a nonzero subspace of $X$. We say that a tuple $\mathcal{T}=\left(T_{1}, T_{2}, \ldots, T_{n}\right)$ is called $M$-transitive with respect to a tuple of nonnegative integer sequences

$$
\left(\left\{k_{j(1)}\right\}_{j},\left\{k_{j(2)}\right\}_{j}, \ldots,\left\{k_{j(n)}\right\}_{j}\right),
$$

if for every nonempty relatively open subsets $U, V$ of $X$ there exists $j_{0} \in \mathbb{N}$ such that $T_{1}^{-k_{j_{0}(1)}} T_{2}^{-k_{j_{0}(2)}} \ldots T_{n}^{-k_{j_{0}(n)}}(U) \cap V$ contains a relatively open nonempty subset of $M$. Also, we say that an n-tuple $\mathcal{T}$ is $M$-transitive if it is $M$-transitive with respect an n-tuple of nonnegative integer sequences.

Suprisingly, there are something that does not happen for single operators. For example, hypercyclic tuples can arise in finite dimensional, and there are operators that have somewhere dense orbits that are not everywhere dense (see [1]). Also, we note that there are subspace-hypercyclic operators that are not hypercyclic (see [2]).

\section{Main Results}

In this section, we investigate subspace-transitivity for tuples of operators.

Theorem 2.1. Suppose that $\mathcal{T}=\left(T_{1}, T_{2}, \ldots, T_{n}\right)$ is an n-tuple of operators acting on a separable infinite dimensional Banach space $X$ over $\mathbf{C}$ and 
$M$ is a nonzero subspace of $X$. Then $\mathcal{T}$ is $M$-transitive if and only if for any nonempty sets $U \subset M$ and $V \subset M$, both relatively open, there exists a tuple $\left(k_{1}, k_{2}, \ldots, k_{n}\right)$ of integers such that $T_{1}^{-k_{1}} T_{2}^{-k_{2}} \ldots T_{n}^{-k_{n}}(U) \cap V$ is nonempty and $T_{1}^{-k_{1}} T_{2}^{-k_{2}} \ldots T_{n}^{-k_{n}} M \subset M$.

Proof. Let $\mathcal{T}$ be $M$-transitive with respect to $M$ and let $U$ and $V$ be nonempty relatively open subsets of $M$. Hence there exists a tuple $\left(k_{1}, k_{2}, \ldots, k_{n}\right)$ of integers such that $T_{1}^{-k_{1}} T_{2}^{-k_{2}} \ldots T_{n}^{-k_{n}}(U) \cap V$ contains a relatively open nonempty set $W$. To show that $T_{1}^{-k_{1}} T_{2}^{-k_{2}} \ldots T_{n}^{-k_{n}} M \subset M$, let $x \in M$ and note that

$$
T_{1}^{k_{1}} T_{2}^{k_{2}} \ldots T_{n}^{k_{n}} W \subset U \cap T_{1}^{k_{1}} T_{2}^{k_{2}} \ldots T_{n}^{k_{n}} V \subset U \subset M .
$$

If $x_{0} \in W$, then there exists $r>0$ small enough such that $x_{0}+r x \in W$, since $W$ is relatively open. Thus

$$
T_{1}^{k_{1}} T_{2}^{k_{2}} \ldots T_{n}^{k_{n}}\left(x_{0}+r x\right)=T_{1}^{k_{1}} T_{2}^{k_{2}} \ldots T_{n}^{k_{n}} x_{0}+r T_{1}^{k_{1}} T_{2}^{k_{2}} \ldots T_{n}^{k_{n}} x \in M,
$$

which implies that $T_{1}^{k_{1}} T_{2}^{k_{2}} \ldots T_{n}^{k_{n}} x \in M$. Thus $T_{1}^{k_{1}} T_{2}^{k_{2}} \ldots T_{n}^{k_{n}} M \subset M$. Conversely, note that $T_{1}^{k_{1}} T_{2}^{k_{2}} \ldots T_{n}^{k_{n}}: M \rightarrow M$ is continuous and so

$$
T_{1}^{-k_{1}} T_{2}^{-k_{2}} \ldots T_{n}^{-k_{n}}(U) \cap V
$$

is relatively open and nonempty subset of $M$. This completes the proof.

Lemma 2.2. Suppose that $\mathcal{T}=\left(T_{1}, T_{2}, \ldots, T_{n}\right)$ is an n-tuple of operators acting on a separable infinite dimensional Banach space $X$ over $\mathbf{C}$ and $M$ is a nonzero subspace of $X$. Suppose that $\mathcal{T}$ is subspace-transitive with respect to $M$. Then $M$ contains a dense $G_{\delta}$ set.

Proof. Let $\left\{B_{n}: n \in \mathbb{N}\right\}$ be a countable open basis for the relative topology of $M$. In Theorem 2.1, put $U=B_{i}$ and $V=B_{j}$, then there exists $K_{i, j}^{m} \in \mathbb{N} \cup\{0\}$ for $m=1, \ldots, n$ satisfying that $T_{1}^{-k_{i, j}^{1}} T_{2}^{-k_{i, j}^{2}} \ldots T_{n}^{-k_{i, j}^{n}}\left(B_{i}\right) \cap B_{j}$ is relatively open. Hence, the sets

$$
G_{i}=\bigcup_{j} T_{1}^{-k_{i, j}^{1}} T_{2}^{-k_{i, j}^{2}} \ldots T_{n}^{-k_{i, j}^{n}}\left(B_{i}\right) \cap B_{j}
$$

are relatively open. Also, each $G_{i}$ is dense since it intersects each relatively open set in $M$. Hence, $\bigcap_{i} G_{i}$ is also dense and the proof is complete.

Corollary 2.3. Under the hypothesis and the notations of Lemma 2.2, the set

$$
\bigcap_{i} \bigcup\left\{T_{1}^{-k_{1}} T_{2}^{-k_{2}} \ldots T_{n}^{-k_{n}}\left(B_{i}\right) \cap M: K_{1}, \ldots, k_{n} \geq 0\right\}
$$


is a dense subset of $M$.

Proof. Since

$$
\bigcap_{i} \bigcup_{j} T_{1}^{-k_{i, j}^{1}} T_{2}^{-k_{i, j}^{2}} \ldots T_{n}^{-k_{i, j}^{n}}\left(B_{i}\right) \cap B_{j}
$$

is a subset of

$$
\bigcap_{i} \bigcup\left\{T_{1}^{-k_{1}} T_{2}^{-k_{2}} \ldots T_{n}^{-k_{n}}\left(B_{i}\right) \cap M: K_{1}, \ldots, k_{n} \geq 0\right\},
$$

it is clear.

Theorem 2.4. Suppose that $\mathcal{T}=\left(T_{1}, T_{2}, \ldots, T_{n}\right)$ is an n-tuple of operators acting on a separable infinite dimensional Banach space $X$ over $\mathbf{C}$ and $M$ is a nonzero subspace of $X$. If $\mathcal{T}$ is $M$-transitive, then $\mathcal{T}$ is $M$-hypercyclic.

Proof. Let $\left\{B_{n}: n \in \mathbb{N}\right\}$ be a countable open basis for the relative topology of $M$. Note that

$$
x \in \bigcap_{i} \bigcup\left\{T_{1}^{-k_{1}} T_{2}^{-k_{2}} \ldots T_{n}^{-k_{n}}\left(B_{i}\right) \cap M: K_{1}, \ldots, k_{n} \geq 0\right\}
$$

if and only if for any integer $j \geq 1$, there exists a tuple $\left(k_{1}, k_{2}, \ldots, k_{n}\right)$ of integers such that $T_{1}^{k_{1}} T_{2}^{k_{2}} \ldots T_{n}^{k_{n}} x \in B_{j}$. This occurs if and only if $\operatorname{Or} b(\mathcal{T}, x) \cap M$ is dense in $M$ or equivalently, if $x \in H C(\mathcal{T}, M)$. Hence

$$
H C(\mathcal{T}, M)=\bigcap_{i} \bigcup\left\{T_{1}^{-k_{1}} T_{2}^{-k_{2}} \ldots T_{n}^{-k_{n}}\left(B_{i}\right) \cap M: K_{1}, \ldots, k_{n} \geq 0\right\} .
$$

Now by Corollary 2.3, the proof is complete.

\section{References}

[1] N.S. Feldman, Hypercyclic tuples of operators and somewhere dense orbits, J. Math. Appl., 346 (2008), 82-98.

[2] B.F. Madore, R.A. Martinez-Avendano, Subsapce hypercyclicity, Journal of Mathematical Analysis and Applications, 375, No. 2 (2011), 502-511. 